Supporting Information

\title{
Morphological evolutions of iron films on PDMS substrates under uniaxial compression/tension
}

Tian-Xiang Gao, Ya-Dong Sun, Yu-Fei Feng and Sen-Jiang Yu*

Department of Physics, China Jiliang University, Hangzhou 310018, P.R. China

Corresponding author: Sen-Jiang Yu (sjyu@cjlu.edu.cn; senjiangyu@hotmail.com) 


\section{Supplementary Figures and captions}
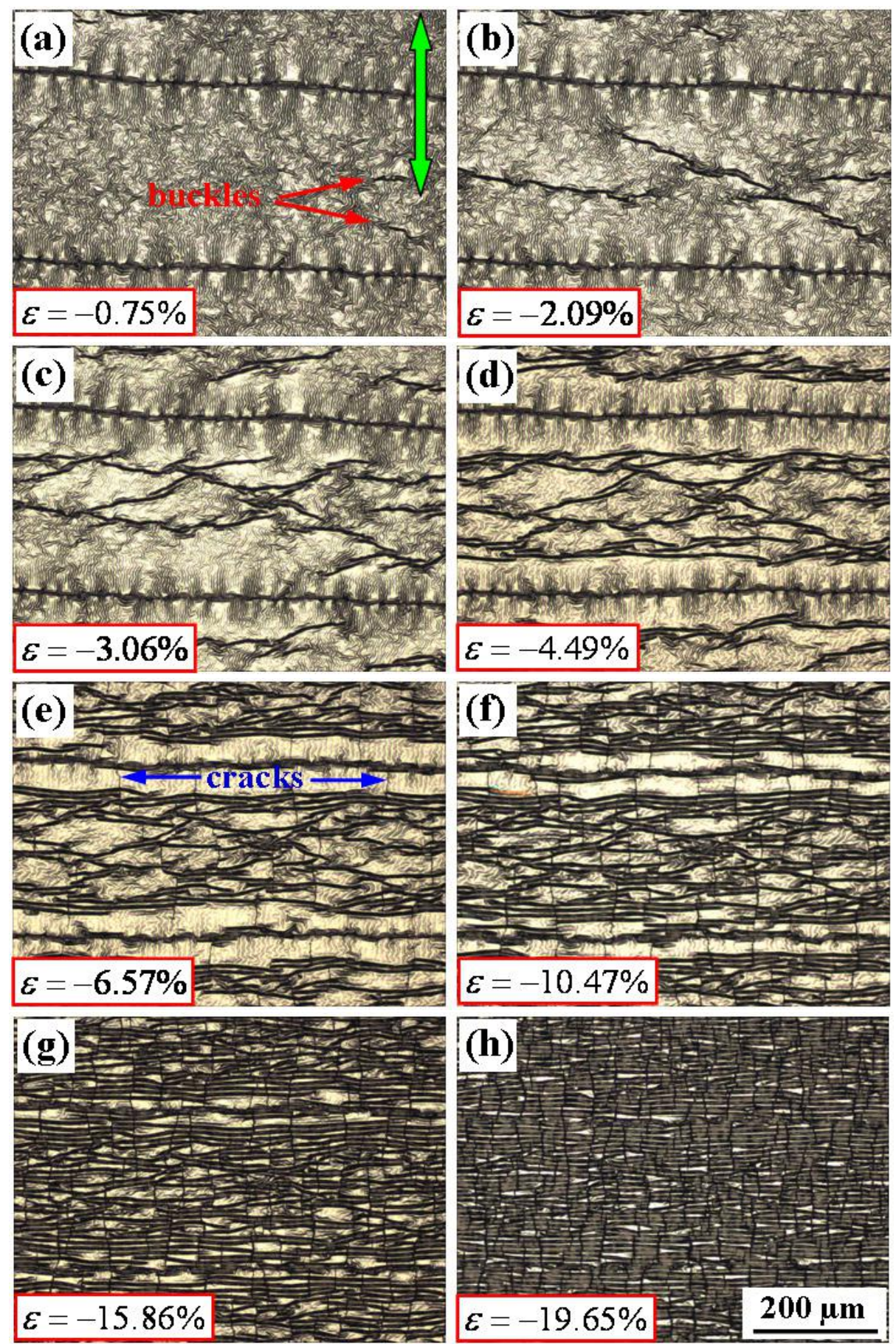

Figure S1. (colour online) In situ morphological evolution of the film with releasing the prestrain of the PDMS. The two-side arrow represents the loading direction of the PDMS. The data appearing in the bottom-left corners represent the longitudinal compressive strain in the film. The red arrows denote the transverse buckles. The blue arrows denote the longitudinal cracks. All images have the same size of $700 \times 525 \mu \mathrm{m}^{2}$. This supplementary figure corresponds to Figure 3 in main text. 

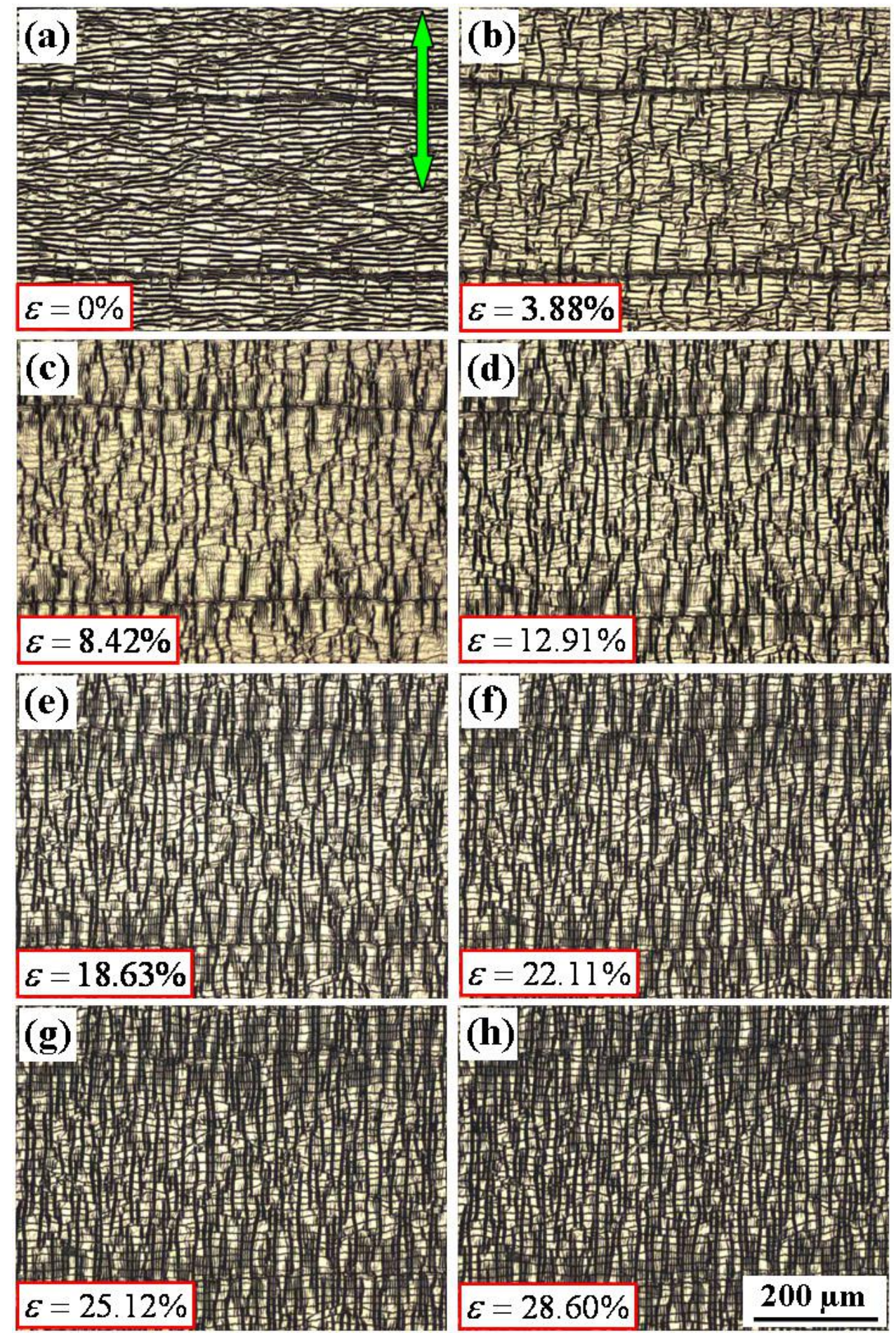

Figure S2. (colour online) In situ morphological evolution of the film with further straining the PDMS. The two-side arrow represents the loading direction of the PDMS. The data appearing in the bottom-left corners represent the longitudinal tensile strain in the film. All images have the same size of $700 \times 525 \mu^{2}$. This supplementary figure corresponds to Figure 4 in main text. 